\title{
A Novel Connection between Rods and ON Cone Bipolar Cells Revealed by Ectopic Metabotropic Glutamate Receptor 7 (mGluR7) in mGluR6-Deficient Mouse Retinas
}

\author{
Yoshihiko Tsukamoto, ${ }^{1}$ Katsuko Morigiwa, ${ }^{3}$ Masaaki Ishii, ${ }^{1,2}$ Motoharu Takao, ${ }^{4}$ Ken Iwatsuki, ${ }^{5}$ Shigetada Nakanishi, ${ }^{6}$ \\ and Yutaka Fukuda ${ }^{7}$ \\ ${ }^{1}$ Departments of Biology and 20phthalmology, Hyogo College of Medicine, Nishinomiya, Hyogo 663-8501, Japan, ${ }^{3}$ EW 89 Synchronicity Research Institute, \\ Tokyo 103-0027, Japan, ${ }^{4}$ Department of Human and Information Science, Tokai University, Hiratsuka, Kanagawa 259-1292, Japan, ${ }^{5}$ Department of \\ Neuroscience, Mount Sinai School of Medicine, New York, New York 10029, ${ }^{6}$ Osaka Bioscience Institute, Suita, Osaka 565-0874, Japan, and ${ }^{7}$ Department of \\ Physiology, Osaka University Graduate School of Medicine, Suita, Osaka 565-0871, Japan
}

\begin{abstract}
Since the discovery of direct chemical synapses between rod photoreceptor and OFF cone bipolar cells in mouse retinas, whether the ON cone bipolar cell also receive direct chemical input from rod has been a pending question. In finding that metabotropic glutamate receptor 7 (mGluR7) was uniquely expressed in dendrites of $\mathrm{ON}$ cone bipolar cells in the mGluR6-deficient mouse retina, we used this ectopic mGluR7 immunoreactivity as a specific marker for the $\mathrm{ON}$ cone bipolar to search for its rod connection. Here, we show that a certain type of $\mathrm{ON}$ cone bipolar cell forms ribbon-associated synapses not only with cones, but also rods. This finding was verified in the wild-type mouse retina by three-dimensional reconstruction of bipolar cells from serial electron micrographs. These $\mathrm{ON}$ cone bipolars were further identified as corresponding to type 7 of mouse bipolar cell described by Ghosh et al. (2004) and also to the green fluorescent protein (GFP)-labeled type 7 bipolars in the $\alpha$-gustducin-GFP transgenic mouse. Our findings suggest that, in mice, rod signals bifurcate into a third ON and OFF pathway in addition to the two known routes to cone bipolar cells: (1) via rod chemical synapse $\rightarrow$ rod bipolar $\rightarrow$ AII amacrine $\rightarrow$ ON and OFF cone bipolar cells; (2) via rod-cone gap junction $\rightarrow$ cone chemical synapse $\rightarrow$ ON and OFF cone bipolar cells; and (3) via rod chemical synapse $\rightarrow \mathrm{ON}$ and OFF cone bipolar cells. This third novel pathway is thought to transmit fast and moderately light-sensitive rod signals, functioning to smooth out the intensity changes at the scotopic-mesopic interface.
\end{abstract}

Key words: cone; glutamate receptor; network; neurotransmission; retina; rod; vision

\section{Introduction}

In the mammalian retina, the two known rod pathways adapting to different light intensities stem from the rod photoreceptor cell terminal (Sterling, 1998; Wässle, 2004). The main pathway [rod $\rightarrow$ rod bipolar $\rightarrow$ AII amacrine cell (via chemical synapse unless stated otherwise), bifurcating onto ON cone bipolar (via gap junction) and OFF cone bipolar cells] functions at the scotopic (pure rod) range of light intensity. The other pathway [rod $\rightarrow$ cone (via gap junction), which bifurcates directly onto $\mathrm{ON}$ and OFF cone bipolar cells] functions at the mesopic (mixed rod and cone) range of light intensity (Sharpe and Stockman, 1999). Both $\mathrm{ON}$ and OFF cone bipolar cells play a major role in these path-

\footnotetext{
Received Dec. 30, 2006; revised April 19, 2007; accepted May 4, 2007.

This work was supported in part by The Strategic Promotion System for Brain Science from the Science and Technology Agency of Japan (K.M.), Grant-in-Aid 12878144 from Japan Society for the Promotion of Science (Y.T.), and a Grant-in-Aid for Researchers, Hyogo College of Medicine (Y.T.). Our sincere thanks are directed to Dr. R. F. Margolskee (Mount Sinai Medical Center, New York, NY) for gifts of GUS-GFP mouse tissues, to Dr. N. Vardi (University of Pennsylvania, Philadelphia, PA) for reading our previous manuscript, to Dr. A. Kaneko (Kio University, Nara, Japan) for comments on ON cone bipolar cells, to Ms. K. Iseki for animal care, and to Ms. N. Omi, Ms S. Inoue, and Ms M. Ueda for assistance.

Correspondence should be addressed to Yoshihiko Tsukamoto, Depatment of Biology, Hyogo College of Medicine, 1-1, Mukogawa, Nishinomiya, Hyogo 663-8501, Japan. E-mail: ytsuka@hyo-med.ac.jp.

D01:10.1523/JNEUROSCI.5646-06.2007

Copyright $\odot 2007$ Society for Neuroscience $\quad$ 0270-6474/07/276261-07\$15.00/0
}

ways. Yet, ever since a third pathway in which rod terminals had direct chemical synapses with certain types of OFF cone bipolar cell has been found in mammalian retinas (Soucy et al., 1998; Hack et al., 1999; Tsukamoto et al., 2001; Li et al., 2004), whether a third rod-ON cone bipolar cell pathway existed as well has remained to be determined.

So far, no direct rod photoreceptor and ON cone bipolar connection had been detected. Synaptic connections in the neuronal microcircuitry can be analyzed with serial electron micrographs, but cytochemical identification of the target cells is always desirable for efficiency and reliability. Based on Tagawa et al.'s (1999) observation, we came upon a candidate marker for ON cone bipolar cells. In their study of the metabotropic glutamate receptor 6 (mGluR6)-deficient mouse retina, they showed mGluR7 immunoreactivity ectopically expressed in the outer plexiform layer (OPL; the dendritic side of bipolar cells). The mGluR7, in the same group III of mGluRs as mGluR6 (Okamoto et al., 1994), generally resides in the inner plexiform layer (IPL) at the axon terminals of ON and OFF cone bipolar cells (Brandstätter et al., 1996). Although the specificity of mGluR7 expression was not determined in their study, it was quite possible that the ectopic mGluR7 was expressed in the dendrites of ON cone bipolar cells in the mGluR6-deficient retina. 
Here, by light and electron microscopic (EM) immunocytochemistry, we confirmed the specific expression of mGluR7 in the dendrites of mGluR6-deficient ON cone bipolar cells. Further, by means of this immunoreactivity, we detected labeled ON cone bipolar dendrites invaginating the rod terminals up to the area apposed to the rod synaptic ribbon in the mGluR6-deficient mouse retina. To ensure that this was not merely an aberrant connection, by three-dimensional reconstruction of $\mathrm{ON}$ cone bipolar cells from serial electron micrographs of the wild-type mouse retina, we verified that type B7 ON cone bipolar cell dendrites indeed formed ribbon-associated synapses with rods as well as cones. This finding was further corroborated in the $\alpha$-gustducin (GUS)-green fluorescent protein (GFP) transgenic mouse retina, in which the same type 7 ON cone bipolar cell, strongly labeled with GFP, had both rod and cone contacts. This novel ON pathway is thought to serve the mesopic range, similarly to its counterpart OFF pathway, thus suggesting a third paired ON and OFF pathway for the transmission of rod signals in mouse retina.

\section{Materials and Methods}

Animals. The mGluR6-deficient mice (hybrid of 129/SvJ by C57BL/6J) were reproduced from the same strain as reported in the previous literature (Tagawa et al., 1999). Adult mGluR6-deficient and wild-type mice were used for electron and fluorescence immunocytochemistry. All experiments were conducted in compliance with the institutional and $\mathrm{Na}-$ tional Institutes of Health guidelines for animal care and treatment. The retinal tissues of GUS-GFP transgenic mice were generously provided by R. Margolskee (Mount Sinai Medical Center, New York, NY) (Huang et al., 2003).

Tissue preparations for immunocytochemistry. Bipolar cells were dissociated from mouse retinas as follows. After deep anesthesia with sodium pentobarbital (45 mg/kg, i.p.), excised pieces of retinas were incubated for $30 \mathrm{~min}$ in a mixture of papain (1.2 U/retina; P3125; Sigma, St. Louis, $\mathrm{MO}$ ), hyaluronidase (2.25 mg/retina; H3506; Sigma), and collagenase (2.25 mg/retina; C2674; Sigma) in Ames' medium (30 ml; A1420; Sigma), and then for another $30 \mathrm{~min}$ without the hyaluronidase and collagenase. Specimens were then treated with protease inhibitor $(10 \%$; L8531; Sigma), followed by DNAase (3\%; D5025;Sigma) in Ames' medium. They were mechanically triturated using a pipette, and a drop of the cell suspension was infused into the interspace (100 $\mu \mathrm{m}$ in width) between the coverslip and poly-L-lysine-coated slide glass. The adhered cells were then stained and rinsed by perfusion of solutions from a pipette at one end of the coverslip absorbed into a filtration paper at the other end.

For light microscopic immunocytochemistry of sections, after deeply anesthetized mice were perfused with a mixture of $4 \%$ paraformaldehyde in cacodylate buffer at $\mathrm{pH} 7.4$, their excised retinas were immersed in the same fixative for $3 \mathrm{~h}$, microwave irradiated for $10 \mathrm{~min}$, rinsed, and cryoprotected in $30 \%$ sucrose in phosphate buffer. Radial sections were cut at the thickness of 12-16 $\mu \mathrm{m}$ with a cryostat (Sakura Finetek, Tokyo, Japan). The samples were observed with a confocal laser scanning microscope (LSM510-16; Zeiss, Oberkochen, Germany) under a 100× objective ( $z$-axis step size, $0.43 \mu \mathrm{m}$ ).

For immunoelectron microscopy, $0.01 \%$ glutaraldehyde was added to the fixative and fixation was applied only for $30 \mathrm{~min}$. Cryosections were made for pre-embedding immunoelectron labeling according to Sawada and Esaki (2002). The specimens embedded in Araldite were cut at the thickness of $90 \mathrm{~nm}$ with an ultramicrotome (Leica, Nussloch, Germany). Ultrathin sections were mounted on formvar-covered slot grids, and photographed with an electron microscope (JEM1220; JEOL, Tokyo, Japan).

Antibodies. Anti-CtBP2 (a transcription factor, C-terminal binding protein 2, identical to B-domain of ribbon-specific RIBEYE protein) (Schmitz et al., 2000) rabbit polyclonal (Peptide Institute, Osaka, Japan), anti-protein kinase $\mathrm{C} \alpha(\mathrm{PKC} \alpha)$ rabbit polyclonal (Sigma), mouse monoclonal (MC5; Amersham Bioscience, Arlington Heights, IL), anti-
mGluR7a rabbit polyclonal (Upstate Biotechnology, Lake Placid, NY), anti-G $\alpha_{\mathrm{o}}$ mouse monoclonal (2A; Millipore, Temecula, CA), and antiGFP mouse monoclonal (Millipore) antibodies were used. For double staining with anti-CtBP2 rabbit polyclonal and anti-mGluR7a rabbit polyclonal antibodies, we used the tyramide signal amplification kit (T20925; Invitrogen, Eugene, OR).

Serial reconstruction. For reconstruction of bipolar cells as shown in Figure 3, we used the same series of electron micrographs of the wild-type mouse retina as that in our previous study (Tsukamoto et al., 2001). Certain synapses were rephotographed at 40,000 $\times$. Three-dimensional images were reconstructed with TRI graphic software (Ratoc, Tokyo, Japan) for Windows NT.

\section{Results}

\section{Ectopic expression of mGluR7 in mGluR6-deficient ON cone bipolar dendrites}

In the wild-type mouse retina, mGluR7 is inherently located at the axon terminals of both ON and OFF cone bipolar cells, but not at their dendrites (Brandstätter et al.,1996). However, Tagawa et al. (1999) indicated mGluR7 immunoreactivity at the OPL in the mGluR6-deficient mouse retina, although the type of cells expressing the mGluR7 was undetermined. Because rod bipolar cells are known to have no mGluR7 in their axons or dendrites, the mGluR7 was most likely expressed in the dendrites of mGluR6-deficient ON cone bipolar cells, and less likely, but possibly, in those of the OFF cone bipolar cells.

To address these possibilities, we first re-examined the findings of Tagawa et al. (1999) and studied the distribution of mGluR7 immunoreactivity in the retinal sections of mGluR6deficient mice. Because no antibody was known to uniquely stain ON cone bipolar cells, we triply stained the sections to discriminate the bipolar types: rod bipolar cells with anti-PKC $\alpha$ antibody in blue, ON cone bipolar and rod bipolar cells with anti-G $\alpha_{\mathrm{o}}$ antibody in green, and receptor protein mGluR7 with antimGluR7 antibody in red (Fig. $1 A-E$ ). Numerous red patches appeared over the IPL (Fig. $1 A$, arrowheads), indicating mGluR7 immunoreactivity of ON and OFF bipolar axon terminals and also some types of amacrine cells. Many red patches were aligned at intervals in the OPL (Fig. $1 A, B, E$, arrows), which coincided with green areas $\left(\mathrm{G} \alpha_{\mathrm{o}}\right)$ but not with blue areas $(\mathrm{PKC} \alpha)$, indicating that the red patches in the OPL immunolabeled the dendrites of ON cone bipolar cells, but not those of rod bipolar cells.

For a more detailed analysis of mGluR7 immunoreactivity in the bipolar cells, we examined dissociated cells from both the wild-type and mGluR6-deficient mice (Fig. $1 F-I$ ). As in the retinal sections, mGluR7 immunoreactivity was evident at the axon terminals of both ON and OFF cone bipolar cells of both wildtype (Fig. $1 F, H$, arrowheads) and mGluR6-deficient (Fig. 1G,I, arrowheads) mouse retinas. The axon terminals of rod bipolar cells were never positive for mGluR7 (data not shown). Identification of the ON and OFF cone bipolar types was made by both mGluR7 and $\mathrm{G} \alpha_{\mathrm{o}}$ immunoreactivities (data not shown) (Dhingra et al., 2000) and by length of the axon processes, which were generally longer for the ON types (Fig. 1, compare $F, G$ with $H, I$ ). Among the five types of known ON cone bipolar cell (Ghosh et al., 2004), the most representative type 6 and type 7 bipolar cells were analyzed (Fig. $1 H, I$ ) (a total of 50 cells from the mGluR6deficient and an equivalent number of cells from the wild-type mouse retina). These type 6 and type 7 cells could not always be discriminated from each other because of irregular deformation, particularly of axonal arbors in the dissociated preparation. Other occasionally detected types of ON cone bipolar cells were eliminated from this analysis. 

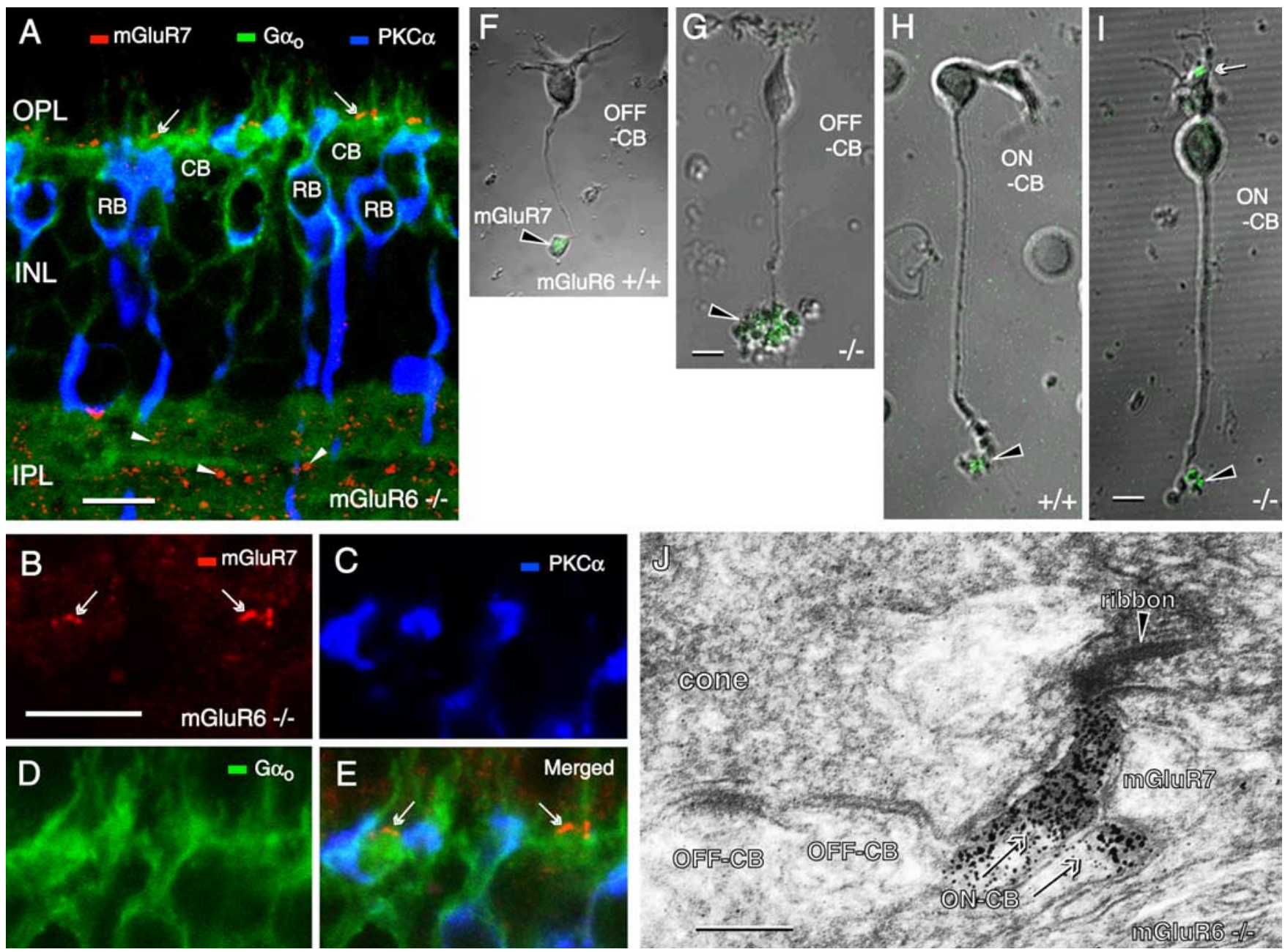

Figure 1. Immunocytochemistry of cone bipolar (CB) cells using the anti-mGluR7 antibody. $\boldsymbol{A}-\boldsymbol{E}$, The $\mathrm{mGluR6}$-deficient mouse retina is triply stained for $\mathrm{mGluR7}$ in red, for $\mathrm{G} \alpha_{0}$ in $\mathrm{green}$ (labeling both ON cone and rod bipolar cells), and for PKC $\alpha$ in blue (labeling only rod bipolar cells). Intermittent red patches in the OPL coincide with green areas (arrows). Numerous red patches (arrowheads) are also scattered in the IPL. Green areas overlap blue areas, but red patches never coincide with any blue areas. $\boldsymbol{F}-\boldsymbol{I}$, Dissociated $0 \mathrm{FF}(\boldsymbol{F}, \boldsymbol{G})$ and $0 \mathrm{~N}(\boldsymbol{H}, \boldsymbol{I})$ cone bipolar cells are immunolabeled for mGluR7 (green) and observed by differential interference contrast. The dendrites are mGluR7-immunoreactive (arrow) only for the $0 \mathrm{~N}$ cone bipolar cell of mGluR6-deficient ( $-I-$ ) mouse (I). The axon terminals are always mGluR7-immunoreactive (arrowheads) for both OFF and ON bipolar cells regardless of the sufficiency $(+/+)$ or deficiency $(-/-$ ) of mGluR6. The dissociated cone bipolar cells appear to correspond to the types of mouse bipolar cells described by Ghosh et al. (2004) as follows: $\boldsymbol{F}$, type 2; $\boldsymbol{G}$, type $3 ; \boldsymbol{H}$, type 6 or 7 ; and $\boldsymbol{I}$, type 6 or 7 .J, Electron micrograph of a cone synaptic terminal in the mGluR6 - / - mouse retina immunolabeled for mGluR7. One immunoreactive dendritic process invaginates the cone pedicle up to a synaptic site facing the cone ribbon. The adjacent smaller immunoreactive process was confirmed to invaginate the cone pedicle in other sections. These two processes are morphologically identified as dendrites of the $0 \mathrm{~N}$ cone bipolar (ON-CB) cells. At the neighboring basal surface of the pedicle, there are two flat synaptic contacts. Their postsynaptic processes are morphologically identified as the dendrites of OFF cone bipolar (OFF-CB) cells, which show no mGluR7 immunoreactivity. Scale bars: $A, B, G, I, 5 \mu \mathrm{m} ; J, 200 \mathrm{~nm}$.

In the wild-type mouse retina, no ectopic expression of mGluR7 was evidenced at any of the dendrites of ON cone bipolar, OFF cone bipolar (Fig. $1 F, H$ ), or rod bipolar cells. In the mGluR6-deficient retina, however, ectopic expression of mGluR7 protein was evident at the dendrites of ON cone bipolar cells (Fig. 1I, arrow), but neither in those of OFF cone bipolar (Fig. 1G) nor rod bipolar cells (data not shown). Thus, the ectopic expression of mGluR7 appeared to be unique to the dendrites of mGluR6-deficient ON cone bipolar cells.

To further confirm the cone bipolar type at the ultrastructural level, we performed EM immunocytochemistry of the mGluR6deficient mouse retina. Bipolar dendrites are known to make two types of synaptic contact, invaginating and flat, at the basal surface of a cone photoreceptor pedicle (Dowling, 1987). The invaginating type of contact is formed between the concave ribbonassociated membrane of a pedicle and the protruding dendritic membranes of ON cone bipolar cells. The flat type is composed of two relatively flat and densified presynaptic and postsynaptic membranes: that of the basal surface of a pedicle and that of the dendritic tips of OFF cone bipolar cells. Significantly, at the basal surface of a pedicle (Fig. $1 \mathrm{~J}$, cone), one dendritic process fully invaginating toward the ribbon (arrowhead) was unequivocally immunoreactive for mGluR7 (Fig.1 J, ON-CB, left arrow). The adjacent smaller dendritic process (ON-CB, right arrow) fully invaginating a pedicle in other sections was also immunoreactive for mGluR7. These were the dendrites of ON cone bipolar cells (Vardi and Morigiwa, 1997), which were definitely different from the semi-invaginating dendrites of certain types of OFF cone bipolar cells (DeVries et al., 2006). In the neighboring area, two flat synaptic contacts with membrane densification (Fig. 1J, OFFCBs) had characteristic postsynaptic processes that were morphologically identified as the dendrites of OFF cone bipolar cells. These OFF cone bipolar dendrites were negative in immunoreactivity for mGluR7, substantiating our finding that the ectopic 

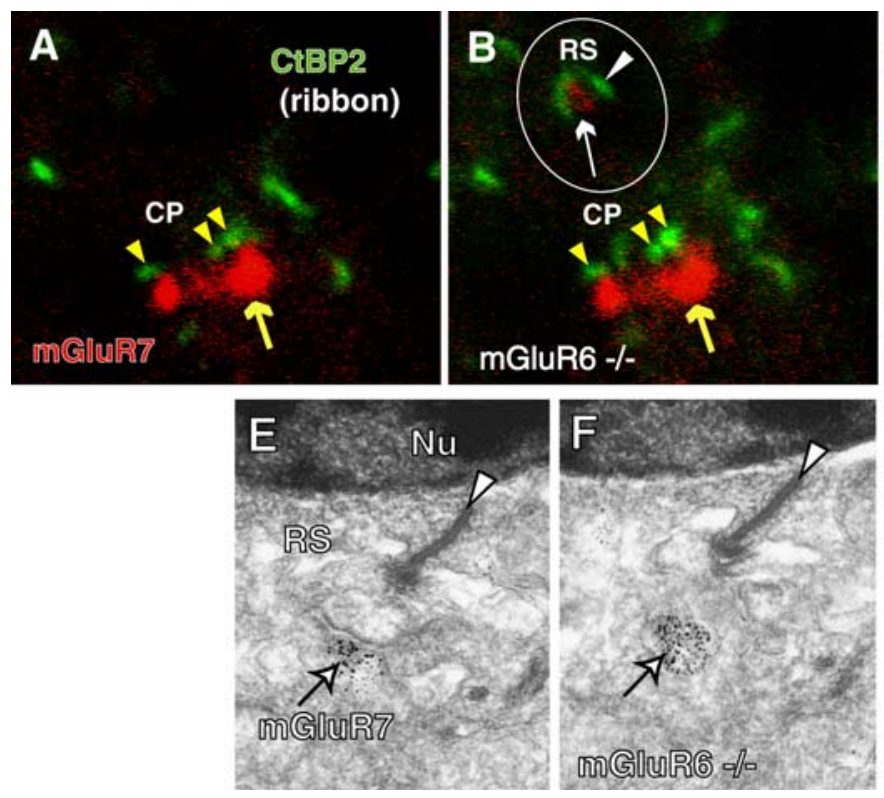
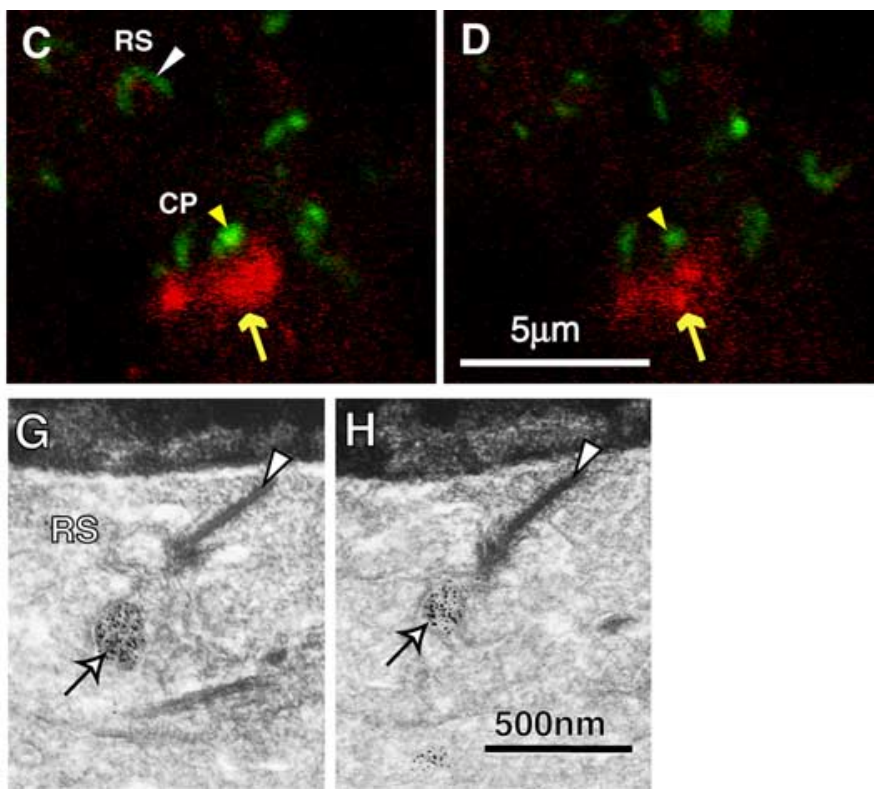

Figure 2. A novel synaptic junction between rod photoreceptor and ON cone bipolar cell in mGluR6-deficient mouse. $A-D$, Rod and cone contacts with mGluR7-labeled ON cone bipolar dendrites (red) in the mGluR6-deficient mouse retina. A ribbon (green) in a rod spherule (RS) is located above a cone pedicle (CP) and shaped like a horseshoe. Ribbons (green) in CPs are relatively small and aligned as clusters. $\boldsymbol{B}, \mathrm{A}$ dendritic tip labeled with mGluR7 immunoreactivity (white arrow) makes contact with the ribbon (white arrowhead) in the RS. A larger dendrite labeled with mGluR7 immunoreactivity (yellow arrow) branches out in apposition to ribbons (yellow arrowheads) in the (P. $\boldsymbol{E}-\boldsymbol{H}$, Electron micrographs of serial sections from the mGluR6 - / - mouse retina immunolabeled for mGluR7. One process is mGluR7-immunoreactive (arrow) and invaginates the rod spherule up to a synaptic ribbon (arrowhead). Nu, The nucleus of a rod photoreceptor cell.

expression of mGluR7 was unique to the ON cone bipolar dendrites of mGluR6-deficient mice.

\section{Rods synaptically connect with one type of ON cone bipolar cell}

Because the ectopic expression of mGluR7 in ON cone bipolar dendrites of mGluR6-deficient mice proved to be a potential probe, we ventured to make use of this specific mGluR7 labeling to address whether synaptic connections might exist between rod photoreceptor and ON cone bipolar cells.

In the mGluR6-deficient mouse retina, we labeled the synaptic ribbons in rod and cone photoreceptors with anti-CtBP2 antibody (in green) and the ON cone bipolar dendrites with antimGluR7 antibody (Fig. $2 A-D$, red). We were able to discriminate the singly present, relatively large horseshoe-shaped synaptic ribbons of rod spherules (Fig. $2 B$, white arrowhead) from those of cone pedicles aligned as clusters of small segments (Fig. $2 A-D$, yellow arrowheads). In close apposition to some of the ribbons in rod spherules, we recognized tiny red patches of mGluR7 immunoreactivity (Fig. $2 \mathrm{~B}$, white arrow) (12 in 17 sections). Much larger mGluR7-labeled red patches of ON cone bipolar dendrites were also observed in apposition to ribbons of most cone pedicles (Fig. $2 A-D$, yellow arrows).

Furthermore, in a series of immunoelectron micrographs of the mGluR6-deficient mouse retina, we occasionally observed mGluR7-immunoreactive dendrites (Fig. $2 E-H$, arrows) (eight in 100 serial ultrathin sections) invaginating the rod spherule up close to the synaptic ribbon (arrowheads). These results indicated that some types of ON cone bipolar cells made ribbon synaptic contacts with rod photoreceptors in the mGluR6-deficient mouse retina.

However, the possibility remained that this rod contact was limited to the mGluR7-positive ON cone bipolar dendrites in the mGluR6-deficient retina. To ensure that this was not an aberrant connectivity caused by mGluR6 deficiency, we undertook a comprehensive search for such morphological connections in serial electron micrographs of the wild-type mouse retina. We reconstructed a total of $11 \mathrm{ON}$ cone bipolar cells in the wild-type mouse retina: four cells for type $\mathrm{B} 5$, three each for types $\mathrm{B} 6$ and $\mathrm{B} 7$, and one for type B8; one of each type (B5-B8) is shown in Figure 3D. The cell types were classified by depth and shape of their axonal arborization based on the morphological data of Ghosh et al. (2004). The axon terminal arbor of these cells spanned levels $40 \sim 90 \%$ of the IPL depth (Fig. 3D).

We found that, of the four ON cone bipolar types (B5-B8) (Fig. 3D), only type B7 extended their dendrites as postsynaptic processes into rod spherules to form ribbon synapses. All three reconstructed type $\mathrm{B} 7$ cells formed ribbon synapses with rods and cones at their dendritic tips. The reconstructed cell 1 , which had three dendritic contacts with rods (circles) and five with cones (triangles), is shown in Figure 3C. Cell 2 had contacts with two rods and five cones, and cell 3 with two rods and seven cones. The invaginating dendrite of type $\mathrm{B} 7$ cone bipolar cell 1 (Fig. $3 A, B$ ) and that of a rod bipolar cell (Fig. $3 A, B$ ) formed parallel ribbon synapses. The axon terminals of the three type B7 bipolar cells were characteristically narrowly stratified at $74 \pm 4 \%(n=3)$ of the IPL depth, at $73 \%$ for the one shown (Fig. 3D). No mixed inputs were observed for the other types of ON cone bipolar cell (B5, B6, B8) in our present series of micrographs.

Because it was known that in the GUS-GFP transgenic mouse (Huang et al., 2003) the type 7 bipolar cell was characteristically labeled with GFP, we examined its rod connectivity in this inherently mGluR6+/+ retina (Fig. 4). Synaptic ribbons of rod and cone photoreceptors (in red) were stained with anti-CtBP2 antibody, and the GFP labeling for type 7 bipolars (in green) was intensified with anti-GFP antibody. In radial sections of three mouse retinas, we observed a total of 70 strongly GFP-labeled type 7 bipolar cells. Synaptic ribbons of rod spherules were dis- 

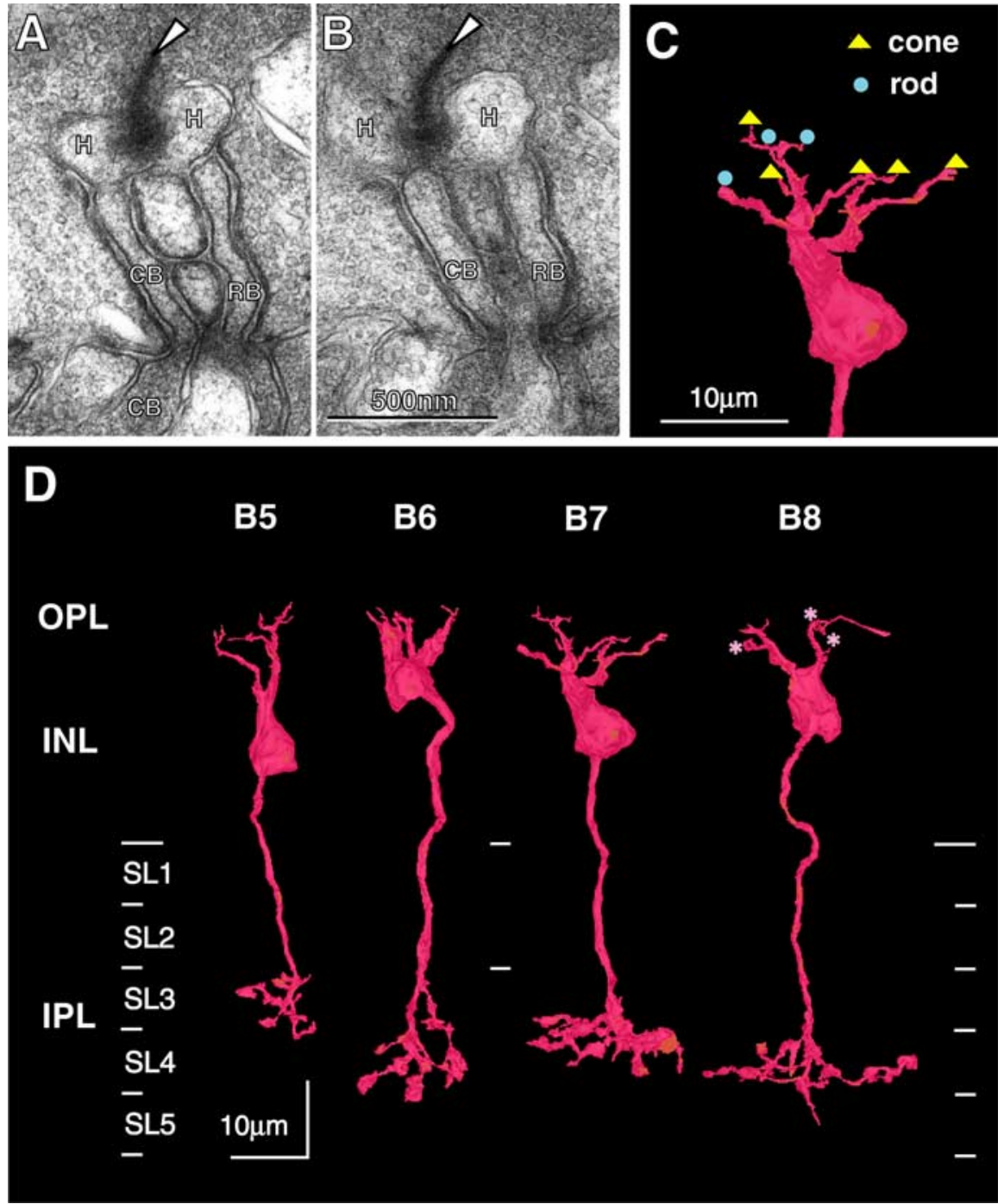

Figure 3. Cone and rod mixed input type of bipolar cell in wild-type mouse. $A, B$, Two adjacent serial electron micrographs of the synaptic terminal of a rod photoreceptor in a wild-type mouse retina, where two invaginating bipolar processes form similar synapses in association with a ribbon (arrowhead). One process comes from a rod bipolar cell (RB) and the other from an ON cone bipolar cell (CB). H, Horizontal cell processes. C, Type B7 ON cone bipolar dendrites have ribbon synapses with rods as well as cones (the reconstructed $B 7$ cell in $\boldsymbol{D}$ at higher magnification). $\boldsymbol{D}$, Reconstruction, from the same serial electron micrographs as in $\boldsymbol{A}$ and $B$, of mouse 0 N cone bipolar cells typed B5, B6, B7, and B8. The depth of the axon terminal in the IPL is $57.8 \pm 6.1 \%(n=4)$ for type B5, $89.0 \pm 4.5 \%(n=3)$ for type B6, $73.5 \pm 4.4 \%(n=3)$ for type B7, and $90.2 \%(n=1)$ for type B8. Asterisks for cell B8 indicate the end points of the series of electron micrographs.

criminated from those of cone pedicles by the aforementioned morphological differences in their shapes, sizes, alignment, and OPL levels. However, as both rod and cone ribbon contacts with the type 7 dendrites were intermingled mostly in the innermost synaptic tier (Fig. 4C,D), in the optical images they often overlapped with each other and prevented clear identification of every rod spherule contact with type 7 bipolar dendrites. Nevertheless, by observing serial optical sections of confocal images with a $100 \times$ objective (Fig. $4 A-D$ ), we were able to serially trace and visualize the three-dimensional configuration of rod-type 7 cone bipolar synaptic junctions (Fig. $4 A-C$, white arrows and arrowheads). Strongly GFP-labeled dendrites in close apposition to rod ribbons were observed (Fig. 4C) approximately once in every two cells (a total of 39 contacts in 70 cells), fourfold less than that observed in electron microscopic reconstruction $(2.3 \pm 0.6$ per cell; $n=3$ ). The synaptic contact between rod photoreceptor and type $7 \mathrm{ON}$ cone bipolar cell thus confirmed in the GUS-GFP transgenic mouse retina, our results demonstrate that a third direct rod-ON cone bipolar pathway exists in mouse retina.

\section{Discussion}

Ectopic mGluR7 expression in mGluR6deficient $\mathrm{ON}$ cone bipolar dendrites In retinas of wild-type mice, mGluR7immunoreactivity is expressed only in the axon terminals of $\mathrm{ON}$ and OFF cone bipolar cells. However, we have shown that, in mGluR6-knock-out mice, it was ectopically expressed in the dendrites of $\mathrm{ON}$ cone bipolar cell as well as in the axon terminals of $\mathrm{ON}$ and OFF cone bipolar cells. mGluR7 is a constituent protein of the presynaptic complex that is synthesized in the soma, transported, and localized in the axon. Yet, Stowell and Craig (1999) showed in cultured hippocampal neurons that mGluR7 was partly transported to the dendrites as well. The dendritic expression of axonal mGluR7 appears to be the result of a fluctuating balance between delivery and exclusion of ectopic proteins within the dendritic domain (our unpublished observation), most likely perturbed in the mGluR6-deficient in vivo mouse retina.

mGluR6 is a $\mathrm{G} \alpha_{\mathrm{o}}$-protein-linked metabotropic glutamate receptor expressed uniquely in the invaginating dendrites of $\mathrm{ON}$ cone and rod bipolar cells. The $\mathrm{G} \alpha_{\mathrm{o}}$ subunit, having a sign-inverting effect, hyperpolarizes the membrane potential $(\mathrm{Na}-$ kanishi, 1994). mGluR6 dysfunction is thus thought to hinder hyperpolarization and cause continuous depolarization of the $\mathrm{ON}$ cone bipolar membrane potential even in the dark. This may elevate axonal transport and proportionately enhance the partial delivery of axonal proteins to dendrites. An excessive dendritic accumulation may overburden the exclusion processes, thus resulting in the ectopic expression of the mGluR7 protein, a consequence of neuronal protein turnover in in vivo dendrites. The underlying mechanism of this ectopic expression is being addressed in our subsequent study.

\section{Rods directly contact ON as well as OFF cone bipolar cells in mouse}

In addition to the two well known pathways, a third direct chemical pathway from rod to OFF cone bipolar cells was most recently established. It was first revealed by electrophysiology of transgenic "coneless" mouse (Soucy et al., 1998), and further substantiated by immunocytochemistry in the rat and mouse (Hack et al., 1999), by three-dimensional reconstruction in the mouse (Tsukamoto et al., 2001), and by confocal microscopy in the rabbit (Li et al., 2004). Here, we report the ON counterpart of this direct rod to OFF cone bipolar synapse in mouse retina. The type of ON cone bipolar cell in our study appears to correspond to type 7 of mouse bipolar cell described by Ghosh et al. (2004) and 

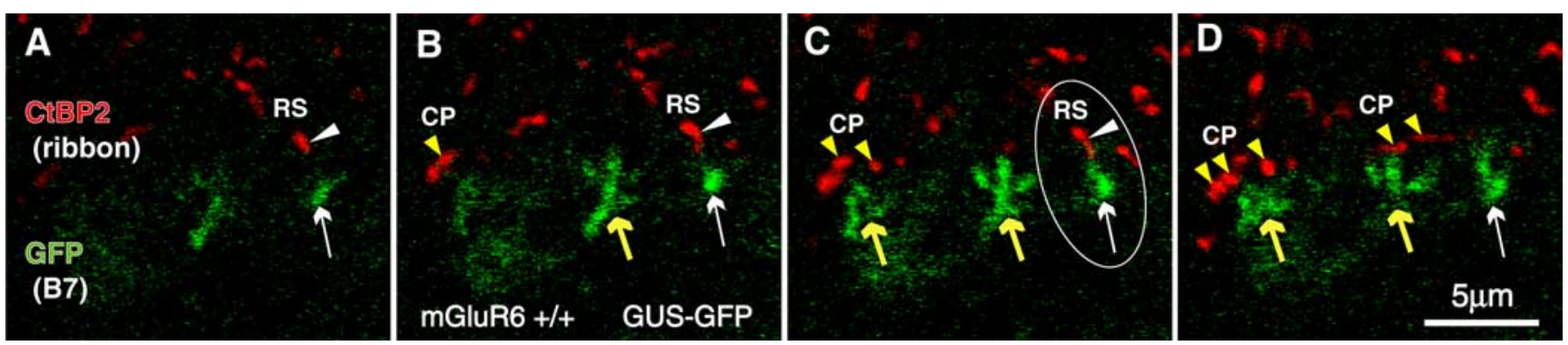

Figure 4. A rod contact and cone contacts with GFP-labeled type 7 bipolar dendrites (green) in the (mGluR6+/+) GUS-GFP mouse retina. $A-C$, A CtBP2-labeled ribbon (red) in a rod spherule (RS) lies singly and spans three optical sections ( $\sim 1.3 \mu \mathrm{m}$ in total thickness). A single dendritic process (white arrow) ascends toward the RS and appears to make contact with the ribbon (white arrowhead). $\boldsymbol{B}-\boldsymbol{D}$, CtBP2-labeled ribbons (red) in cone pedicles (CPs) are relatively small and aligned in clusters. Two dendrites (yellow arrows) branch out in close apposition to ribbons (yellow arrowheads) in (Ps.

also to type CB4a or CB4b described by Pignatelli and Strettoi (2004). This type may also correspond to the bipolar cells that synapse with both ON and ON/OFF types of ganglion cells, as observed by GFP confocal microscopy in mouse retina (Lin and Masland, 2005).

In our previous study of the mouse retinal circuitry focused mainly on the OFF cone bipolar cells (Tsukamoto et al., 2001), the $\mathrm{B}_{\mathrm{ON}}$ cone bipolar cell was shown to have no connection with rods. The difficulty in finding rod-ON cone bipolar contacts then was mainly attributable to the morphological features of the thin bipolar dendrite threading toward a rod spherule, too obscure at places to be continuously traced through serial micrographs. Detecting the continuity between the distal invaginating tip and the proximal ascending dendrite of the bipolar cell often requires tracing through serial electron micrographs of all the surrounding processes in addition to the dendrite under investigation. In the present study, similar to the incentive of Soucy et al.'s (1998) electrophysiological study for the OFF cone bipolar cell, the breakthrough for the $\mathrm{ON}$ cone bipolar cell came with the finding of the specific mGluR7 labeling of mGluR6-deficient ON cone bipolar dendrites. Subsequent comprehensive examination of the same serial electron micrographs enabled us to detect rod contacts of the reconstructed type B7 ON cone bipolar cells, of which cell 3 was the previous $\mathrm{B}_{\mathrm{ON}}$ cone bipolar cell.

Our corroborative finding in the GUS-GFP transgenic mouse retina, which specifically labels type 7 ON cone bipolar cells, posed similar difficulties. The task of discriminating overlapping images of rods and cones in the innermost OPL tier for isolated rod contacts required careful "three dimensionally imaged" tracing of the immunolabeled ribbons and dendrites in serial optical sections of the confocal image (Fig. 4). The discrepancy of our results with those of Haverkamp et al. (2006), which showed GFP-labeled ON cone bipolar dendrites exclusively connected with cone pedicles in the same GUS-GFP mouse strain, may be partly caused by the sparsity of the rod contacts intermingled with those of the cones in the same innermost synaptic tier.

\section{The third ON and OFF rod pathway functions at the mesopic-scotopic interface}

It appears from the present study that this rod-type B7 cone bipolar pathway is sparsely distributed and small in coverage compared with the other two conventional pathways densely extended over the retina. In the first pathway via rod bipolar and AII amacrine cells, our preliminary observation suggests that at least three types (B5-B7) of ON cone bipolar cell are involved in gap junctional connection with AII amacrine cells (our unpublished observation). The second pathway via rod-cone gap junctions covers the entire OPL of the retina. As for the third pathway, it has been shown in our previous study (Tsukamoto et al., 2001) that each rod diverges via gap junctions to other rods forming local syncytia. Thus, similar to the OFF pathway, by pooling a large number of rod signals within each syncytium to the rods that have direct chemical synapses with type B7 bipolar dendrites, the ON pathway may cover a larger area than that directly covered by the sparsely distributed chemical synapses.

What levels of light intensity then might this direct rod-ON cone bipolar pathway serve as opposed to the other two conventional ON pathways? First, it is well established that the rod bipolar to AII amacrine pathway serve low-light levels of scotopic vision. Synaptic amplification of rod signals via numerous ribbon synapses at the rod bipolar axon terminal and noise reduction via the electrical coupling network of AII amacrine cells both contribute to the augmented light sensitivity of this rod bipolar pathway (Vardi and Smith, 1996). In the case of the rod-cone gap junction pathway, which is involved in medium light levels of mesopic vision, more photons for transmission of rod signals is required than that for scotopic vision. Only part of the photoresponse energy of an excited rod is transmitted to cones and no signal amplification occurs via electrical coupling per se in this gap junctional pathway. Because the electrical capacity of a cone terminal is greater than that of a rod terminal, the formation of a reliable rod signal in the cone terminal requires inputs from many excited rods.

As for the direct rod-ON cone bipolar pathway, the photoresponse of an excited rod is transmitted via a chemical synapse. The rod-rod bipolar synaptic signal transfer is known to be similar to a nonlinearly amplified digital gate (Sampath and Rieke, 2004). Thus, when a particular group of rods in this third pathway is excited by photons, their outputs to ON cone bipolar cells should similarly be amplified, allowing the pathway to be more light sensitive than the rod-cone electrical coupling pathway and yet less light-sensitive than the rod bipolar to AII pathway. Hence, this third pathway may possibly serve to smooth out the intensity changes at the boundary between the scotopic and mesopic levels. In fact, Pang et al. (2004) have recorded responses to mixed rod and cone inputs from one type of bipolar cell whose axon terminals ramified at the depth of $70-85 \%$ (ON sublamina) of the IPL in the dark-adapted mouse retina. They noted that rod signals in these bipolars were highly amplified and effectively transmitted to the cone system. Their results appear to correspond with the characteristics of the rod-driven ON cone bipolar type discovered in our study, although the contribution of the conventional pathways cannot be ruled out.

It appears that, in rod pathways, ON and OFF systems form 
dual communication channels for the coding and transmission of rod signals to cone bipolar cells, adapting them to bidirectional changes in illumination from the mean level of three different light intensities. This triplet rod circuitry provides for different light sensitivities: via rod bipolar to AII amacrine cells at the scotopic level, via rod-cone gap junctions at the mesopic level, and via rod chemical synapse at the scotopic-mesopic interface. The third rod-driven chemical pathway may function during the scotopic-mesopic transition by continuously feeding visual signals to the cone bipolar cell as animals move between dimly and moderately lit places. The rod-and-cone mixed-input type of bipolar cells, well known in fish retinas (Stell et al., 1977), enable the transmission of both rod and cone signals via a common bipolar route. Although it is possible that this third ON and OFF roddriven pathway is uniquely developed in mouse, it may be one of the most fundamental designs of retinal circuitry in vertebrates. Whether other mammals with the third OFF pathway have the counterpart ON pathway, and whether other mammalian retinas in general have this triplicity in the rod signaling circuitry are pending questions for future study.

\section{References}

Brandstätter JH, Koulen P, Kuhn R, van der Putten H, Wässle H (1996) Compartmental localization of a metabotropic glutamate receptor (mGluR7): two different active sites at a retinal synapse. J Neurosci 16:4749-4756.

DeVries SH, Li W, Saszik S (2006) Parallel processing in two transmitter microenvironments at the cone photoreceptor synapse. Neuron 50:735-748.

Dhingra A, Lyubarsky A, Jiang M, Pugh Jr EN, Birnbaumer L, Sterling P, Vardi N (2000) The light response of ON bipolar neurons requires $\mathrm{G} \alpha_{\mathrm{o}}$. J Neurosci 20:9053-9058.

Dowling JE (1987) The retina. Cambridge, MA: Harvard UP.

Ghosh KK, Bujan S, Haverkamp S, Feigenspan A, Wässle H (2004) Types of bipolar cells in the mouse retina. J Comp Neurol 469:70-82.

Hack I, Peichl L, Brandstätter JH (1999) An alternative pathway for rod signals in the rodent retina: rod photoreceptors, cone bipolar cells, and the localization of glutamate receptors. Proc Natl Acad Sci USA 96:14130-14135.

Haverkamp S, Michalakis S, Claes E, Seeliger MW, Humphries P, Biel M, Feigenspan A (2006) Synaptic plasticity in $\mathrm{CNGA3}^{-1-}$ mice: cone bipolar cells react on the missing cone input and form ectopic synapses with rods. J Neurosci 26:5248-5255.

Huang L, Max M, Margolskee RF, Su H, Masland RH, Euler T (2003) G protein subunit $\mathrm{G}$ gamma 13 is coexpressed with $\mathrm{G}$ alpha o, $\mathrm{G}$ beta 3 , and G beta 4 in retinal ON bipolar cells. J Comp Neurol 455:1-10.

Li W, Keung JW, Massey SC (2004) Direct synaptic connections between rods and OFF cone bipolar cells in the rabbit retina. J Comp Neurol 474:1-12.

Lin B, Masland RH (2005) Synaptic contacts between an identified type of ON cone bipolar cell and ganglion cells in the mouse retina. Eur J Neurosci 21:1257-1270.

Nakanishi S (1994) Metabotropic glutamate receptors: synaptic transmission, modulation, and plasticity. Neuron 13:1031-1037.

Okamoto N, Hori S, Akazawa C, Hayashi Y, Shigemoto R, Mizuno N, Nakanishi S (1994) Molecular characterization of a new metabotropic glutamate receptor mGluR7 coupled to inhibitory cyclic AMP signal transduction. J Biol Chem 269:1231-1236.

Pang JJ, Gao F, Wu SM (2004) Light-evoked current responses in rod bipolar cells, cone depolarizing bipolar cells and AII amacrine cells in darkadapted mouse retina. J Physiol (Lond) 558:897-912.

Pignatelli V, Strettoi E (2004) Bipolar cells of the mouse retina: a gene gun, morphological study. J Comp Neurol 476:254-266.

Sampath AP, Rieke F (2004) Selective transmission of single photon responses by saturation at the rod-to-rod bipolar synapse. Neuron 41:431-443.

Sawada H, Esaki M (2002) Pre-embedding immunoelectron microscopy with nanogold immunolabeling, silver enhancement, and its stabilization by gold. In: Gold and silver staining: techniques in molecular morphology (Hacker GW, Gu J, ed), pp 169-176. Boca Raton, FL: CRC.

Schmitz F, Konigstorfer A, Sudhof TC (2000) RIBEYE, a component of synaptic ribbons: a protein's journey through evolution provides insight into synaptic ribbon function. Neuron 28:857-872.

Sharpe LT, Stockman A (1999) Rod pathways: the importance of seeing nothing. Trends Neurosci 22:497-504.

Soucy E, Wang Y, Nirenberg S, Nathans J, Meister M (1998) A novel signaling pathway from rod photoreceptors to ganglion cells in mammalian retina. Neuron 21:481-493.

Stell WK, Ishida AT, Lightfoot DO (1977) Structural basis for on-and offcenter responses in retinal bipolar cells. Science 198:1269-1271.

Sterling P (1998) Retina. In: The synaptic organization of the brain (Shepherd GM, ed), pp 205-253. New York: Oxford UP.

Stowell JN, Craig AM (1999) Axon/dendrite targeting of metabotropic glutamate receptors by their cytoplasmic carboxy-terminal domains. Neuron 22:525-536.

Tagawa Y, Sawai H, Ueda Y, Tauchi M, Nakanishi S (1999) Immunohistological studies of metabotropic glutamate receptor subtype 6-deficient mice show no abnormality of retinal cell organization and ganglion cell maturation. J Neurosci 19:2568-2579.

Tsukamoto Y, Morigiwa K, Ueda M, Sterling P (2001) Microcircuits for night vision in mouse retina. J Neurosci 21:8616-8623.

Vardi N, Morigiwa K (1997) ON cone bipolar cells in rat express the metabotropic receptor mGluR6. Vis Neurosci 14:789-794.

Vardi N, Smith RG (1996) The AII amacrine network: coupling can increase correlated activity. Vision Res 36:3743-3757.

Wässle H (2004) Parallel processing in the mammalian retina. Nat Rev Neurosci 5:747-757. 